Revue d'histoire de l'Amérique française

REVUE D.HISTOIRE DE L'AMÉRIQUE FRANÇAISE

\title{
L'Archevêque de Rouen, l'Église du Canada et les historiens, un exemple de déformation historique
}

\section{Jean Blain}

Volume 21, numéro 2, septembre 1967

URI : https://id.erudit.org/iderudit/302668ar

DOI : https://doi.org/10.7202/302668ar

Aller au sommaire du numéro

Éditeur(s)

Institut d'histoire de l'Amérique française

ISSN

0035-2357 (imprimé)

1492-1383 (numérique)

Découvrir la revue

Citer cet article

Blain, J. (1967). L'Archevêque de Rouen, l'Église du Canada et les historiens, un exemple de déformation historique. Revue d'histoire de l'Amérique française, 21(2), 199-216. https://doi.org/10.7202/302668ar d'utilisation que vous pouvez consulter en ligne. 


\section{L'ARCHEVÊUE DE ROUEN, L'ÉGLISE DU CANADA ET LES HISTORIENS, UN EXEMPLE DE DÉFORMATION HISTORIQUE ${ }^{1}$}

Je ne suis pas de ceux qui vouent un culte immodéré à la déesse objectivité en histoire. Je pense que le donné brut lorsqu'il a été usiné dans cette machine infiniment complexe qui s'appelle le cerveau d'un historien en ressort non pas méconnaissable mais artistement poli, subtilement maquillé, parfois légèrement ciselé, pour ne pas dire découpé, toujours adroitement orienté pour qu'il s'insère parfaitement dans une vision du passé qui tient beaucoup de l'analyse de la réalité extramentale, mais qui tient encore plus d'une optique fondamentale chez l'historien, où le type d'intelligence, les prédispositions innées, l'orientation des années de formation, la philosophie de la vie sans compter l'empreinte du milieu et bien d'autres facteurs, ont joué des rôles décisifs quoique difficiles à départager. L'historien s'approche de l'idéal s'il peut rendre sa vision assez souple pour que le donné brut ait autant de chances de la modifier que celle-ci peut en avoir de l'assumer quand elle lui fait subir le raffinage commode de la compréhension.

Tout cela pour expliquer que si je parle de déformations à propos de certains historiens du passé, je n'entends nullement confronter ces déformations avec une sacro-sainte vérité objective, mais plutôt avec une vision différente des choses dont la caractéristique principale serait qu'elle s'insère plus aisément dans notre optique de 1967, conscient bien entendu que des recherches historiques futures et de nouvelles orientations de la lorgnette humaine dont le résultat m'apparaîtrait aujourd'hui

1 Conférence prononcée à l'inauguration du Centre de Recherche en Histoire religieuse du Canada, à Ottawa, le jeudi 6 avril 1967.

[ 199 ] 
apocalyptique, risquent fort de me faire subir le sort que je réserve à certains de mes devanciers.

Mais d'abord qui sont ces devanciers? Tous ceux qui de Bertrand de La Tour à Émile Bégin en passant par ce sommet que représente Auguste Gosselin se sont astreints à raconter les débuts de l'Église du Canada. La thèse dont je parle s'est figée

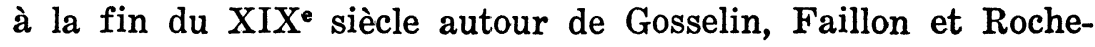
monteix et, pour l'essentiel, s'est transmise jusqu'à nos jours chez ceux qui d'une façon directe ou incidente ont abordé les problèmes religieux du $17^{\circ}$ siècle en Nouvelle-France.

Et que peut-on reprocher à cette thèse? A première vue, c'est de faire un sort peu enviable et peu charitable à Messeigneurs de Harlay, oncle et neveu, qui se sont succédé sur le trône de l'archevêché de Rouen, de leur refuser une place même petite au panthéon des fondateurs de l'Église canadienne, et de nous les donner comme des suppôts hargneux de la monstrueuse erreur gallicane.

$\mathrm{Au}$ vrai, nos deux personnages s'inscrivent dans notre histoire traditionnelle sous le signe de l'encombrement. Rarement aura-t-on pu voir pareils empêcheurs de tourner - ou d'évangéliser - en rond. Et tenaces avec ça; ils ne lâcheront prise qu'en 1674 au moment où sera créé l'évêché de Québec.

L'oncle s'est d'abord insinué en douce au Canada. Comment et à quel moment précis? Nos historiens ont eu peine à le découvrir. Certains font remonter l'affaire à 1632, disant que selon un usage établi, les premiers missionnaires avaient pris à leur port d'embarquement des pouvoirs de l'évêque du lieu et que cette habitude avait amené Mgr de Harlay à considérer le Canada comme faisant partie de son diocèse. ${ }^{2}$ A quoi d'autres historiens répondent que l'explication est invraisemblable puisque les Jésuites qui vinrent au Canada à partir de 1632 détenaient de Rome des patentes officielles en bonne et due forme, et qu'ainsi mandatés par la plus haute autorité, il eût

2 Bertrand de La Tour, Mémoires sur la vie de M. de Laval (Cologne, 1761), 16-17. - E.-M. Faillon, Histoire de la colonie française en Canada (3 vol., Ville-Marie, 1865-1866), 1: 280. 
été absurde qu'ils sollicitent des pouvoirs d'un archevêque de France. $^{3}$

Toutefois, en 1639, le doute n'est plus possible, Mgr de Harlay est bel et bien dans la place. Les Hospitalières de Dieppe qui viennent fonder l'Hôtel-Dieu de Québec lui servent de cheval de Troie. L'autorisation qu'il leur donne stipule qu'elles resteront sous ses ordres jusqu'à ce que soit établie la hiérarchie épiscopale au Canada. Pendant sept ans, Mgr de Harlay déléguera sa juridiction sur les Hospitalières au supérieur des Jésuites de Québec que l'annaliste de l'Hôtel-Dieu en 1646 finit par désigner sous le titre de "grand Vicaire de Monseigneur l'archevesque de Rouen, notre prélat et supérieur".4

Chose étrange, ces faits chez nos historiens de l'Église, n'ont pas reçu l'attention qu'ils méritent. Dans les discussions visant à expliquer ce qu'on nomme les "prétentions" de l'archevêque de Rouen, on n'en fait guère mention et on s'acharne plutôt contre l'argument plus faible selon lequel le prélat aurait accordé quelque vague juridiction à des missionnaires en partance pour le Canada. ${ }^{5}$

Quoi qu'il en soit de 1639 à 1646 les dégâts sont fort circonscrits et ne débordent pas les murs de l'Hôtel-Dieu de Québec. C'est en 1647 que les choses commencent à mal aller. Cette année-là, on trouve dans le Journal des Jésuites cette annotation assez obscure: "Je proposai - c'est le supérieur des Jésuites qui parle - le voyage de France d'un de nos pères pour les affaires des Ursulines, Hospitalières, Iroquois et validité des

${ }^{3}$ Auguste Gosselin, Juridiction exercée par l'archevêque de Rouen (Evreux, 1895) 22. - C. de Rochemonteix, Les Jésuites et la NouvelleFrance au XVIIe siècle (3 vol., Paris, 1895), 2: 190-193.

4 Voir: Obédience de Monseigneur l'Archevêque de Rouen, 20 mars 1639. Obédience donnée par M. Heaume, directeur de l'Hôtel-Dieu de Dieppe, 1639. Obédience du 24 mars 1640. Archives de l'Hôtel-Dieu de Québec, [AHD-Q], T. 21. Voir aussi dans AHD-Q, Annales, 53 et Chroniques de l'Hôtel-Dieu de Québec, Reg 2 : 1-2.

5 Dans La Mission du Canada avant Mgr de Laval (Evreux, 1909) publié quatorze ans après Juridiction exercée par l'archevêque de Rouen, Gosselin minimise le rôle joué par Mgr de Harlay avant 1647 qu'il avait pourtant bien décrit dans son ouvrage précédent. 
sacrements de mariage qu'on nous disputait par des lettres venues de France cette année." ${ }^{6}$

Les Jésuites laisseront passer six ans avant d'éclairer notre chandelle. Le 6 août 1653, on note dans leur Journal:

[II] est à remarquer que n'y ayant eu rapport à aucun évêque pour le gouvernement spirituel de ce pays jusqu'en l'an 1647, il fut pour lors considéré à l'occasion des vestures et professions des religieuses qu'on ne pouvait s'en passer, et la susdite année, le P. Vimont passant en France fut surtout chargé de cette affaire pour l'assurance des professions des religieuses. Le $\mathrm{P}$. Vimont après avoir consulté Rome, les principaux Pères de notre compagnie de la maison-professe et du collège, le sens plus commun fut qu'il fallait s'adresser et attacher à $M$. de Rouen; Ensuite le P. Vimont s'adressa au P. Pingeolet, pour lors recteur du collège de Rouen par la faveur et assistance duquel on obtint de M. l'Árchevêque de Rouen, l'ancien, lettre de grands vicaires; ce qui étant apporté ici avec les lettres et résolutions de tous nos pères confirmatives de ce que dessus, on procéda avec assurance à recevoir les professions des religieuses. On ne jugea pas toutefois à propos de faire encore éclater beaucoup au dehors cette affaire. ${ }^{7}$

Ces textes suscitèrent les commentaires de nos historiens qui au point de départ sans autre source que celle que nous venons de citer déclarent que le responsable direct ou indirect des lettres qui mettaient en doute la compétence des Jésuites à recevoir les professions religieuses n'était nul autre que $\mathbf{M g r}$ de Harlay lui-même, tant il espérait étendre sa juridiction sur la colonie.

Rochemonteix, l'historien des Jésuites, écrit: “... En 1647, des lettres venues de France leur apprirent que Mgr l'archevêque de Rouen prétendait avoir droit de juridiction sur les pays de l'Amérique septentrionale... Aussi très grand fut l'étonne-

- Le Journal des Jésuites (Québec, 1871), 93.

7 Ibid, 186. 
ment des missionnaires... L'inquiétude vint bientôt se mêler à la surprise, et cela se comprend." 8

Pour sa part, Mgr Gosselin raconte la chose comme suit: "De son côté, l'archevêque de Rouen faisait valoir avec habileté et persévérance les titres de sa prétendue juridiction; il réussissait même par des lettres évidemment écrites à son intention à inspirer aux Jésuites du Canada des inquiétudes sérieuses." 9

Il s'agit donc de montrer que pris d'inquiétudes sérieuses les Jésuites agiront à leur corps défendant devant les menées impérialistes de l'archevêque de Rouen. Il sera plus facile ensuite de les décrire comme forcés et honteux à la fois d'accepter une juridiction qu'on leur impose et dont ils n'ont que faire, puisqu'ils ont celle de Rome. Malheureusement, cette explication ne va pas de soi. D'abord, si les lettres de France qui mettent en doute l'autorité des Jésuites ont été inspirées par un Mgr de Harlay ardemment désireux d'étendre son empire gallican aux rives du St-Laurent, comment comprendre que les Jésuites aient dû solliciter l'entremise de leur confrère, le père Pingeolet, recteur du collège de Rouen, pour obtenir des lettres de grand vicaire ? Comment surtout expliquer que cet archevêque qu'on nous donne comme un intrigant, rongé par le désir de voir son autorité reconnue, ait accepté le plus simplement du monde, qu'on ne fasse pas beaucoup éclater l'affaire au dehors, pour reprendre l'expression des Jésuites. Jusqu'à sa mort survenue en 1653, Mgr de Harlay, l'aîné, n'aura pas une seule fois le plaisir de voir proclamer la juridiction qu'il exerce officiellement depuis cinq ans sur la Nouvelle-France. Il faut admettre que ce Harlay de nos historiens est un beau mélange d'ambition et d'humilité démesurées.

Avec l'assurance de ces convictions ultramontaines le père Rochemonteix déclare pour rendre compte du silence qui entoure la juridiction de Rouen: "Il faut croire que les missionnaires ne se montrèrent pas très fiers de leur nouvelle dignité ..." 10

8 Rochemonteix, op. cit, $2: 202$ et 205.

${ }^{9}$ Gosselin, La Mission du Canada avant Mgr de Laval, 103.

10 Rochemonteix, op. cit., 2 : 208. 
Mais fiers ou pas, cette dignité, ils devront l'afficher à partir de 1653. Car Harlay-Champvallon, le neveu qui succède à son oncle, a hérité du trône épiscopal, mais non pas de l'obligeant effacement qui caractérisait son parent. Déjà à titre de coadjuteur, il avait fait savoir de ses nouvelles aux Jésuites de Québec. Puis devenu évêque en titre, il renouvelle les pouvoirs de grand vicaire de leur supérieur. ${ }^{11}$

Cette entrée en scène du neveu oblige sans doute à lever un peu le voile du secret. Le supérieur des Jésuites commence par se donner le titre de "grand vicaire de Mgr l'archevêque de Rouen" dans les registres de la paroisse de Québec. Puis la chose éclate au grand jour, le 15 août 1653, alors que la juridiction de Rouen est proclamée "en la présence de Monsieur le gouverneur et de tout le peuple assemblé pendant la grand'messe". Mgr de Harlay-Champvallon a expédié aux Jésuites un mandement leur enjoignant de publier un jubilé qui ne peut être gagné par les fidèles que par l'intermédiaire de l'Ordinaire. Le grand vicaire est forcé de s'exécuter. ${ }^{12}$

Tout cela, bien entendu, répugne aux Jésuites pour la seule raison qu'ils n'aiment pas servir deux maîtres. Mais il faut avouer que jusqu'en 1657 ils paraissent surmonter leur répugnance avec assez de facilité.

Cette année-là, c'est un autre personnage encombrant qui fait son apparition. Il s'agit de Gabriel de Queylus, supérieur des Messieurs de Saint-Sulpice qui viennent s'établir à Montréal. Monsieur de Queylus a reçu de Mgr de Harlay des pouvoirs de grand vicaire qui lui donne autorité sur toute l'organisation religieuse de la Nouvelle-France. ${ }^{13}$

Il faut voir avec quelles précautions méthodologiques, l'historien Faillon nous raconte la chose. Sulpicien lui-même, l'abbé Faillon est naturellement porté à défendre ses confrères, mais le mieux qu'il puisse faire en l'occurrence c'est encore de montrer

${ }^{11}$ Le Journal des Jésuites, 187.

12 Ibid, 185-187.

13 Rochemonteix, op. cit., 2. - Pièce justificative X, 477, Archives de l'Archevêché de Québec [AAQ] Registre A, feuille détachée. 
que la juridiction de Rouen était un pis-aller inévitable. Pour cela, il nous explique que le pape avait à toutes fins pratiques refusé en 1643 d'autoriser le nonce apostolique à donner juridiction aux ecclésiastiques destinés à Montréal. Force fut donc aux Sulpiciens de s'adresser à Rouen puisque, se hâte-t-il de préciser, c'est de là que "les Pères Jésuites recevaient eux-mêmes [la juridiction] qu'ils exerçaient dans la Nouvelle-France". Faillon va même jusqu'à tenter d'établir, en déformant le sens d'une expression latine, que les titres d'official et de grand vicaire ont été attribués à Monsieur de Queylus par décision unilatérale de $\mathrm{Mgr}$ de Harlay et sans que le sulpicien en fasse la demande. ${ }^{14}$

Vraiment, au dire de nos historiens, l'investiture de Mgr de Rouen n'était pas une dignité qu'on arborait avec fierté en Nouvelle-France. Malgré tout Jésuites et Sulpiciens ne firent pas faute de se la disputer. Au début, M. de Queylus fut roi et maître à Québec comme à Montréal. Mais les Jésuites qui avaient pourtant trouvé là l'occasion rêvée de se débarrasser des liens gênants qui les rattachaient à Rouen, goûtèrent assez peu, semble-t-il, de recevoir leurs ordres de M. de Queylus. Ils s'en ouvrirent à leur général, qui à son tour révéla la chose au confesseur du roi. Finalement Mgr de Harlay qui connaissait bien son Salomon décida de limiter la juridiction de M. de Queylus à l'île de Montréal et rétablit celle du supérieur des Jésuites sur la région de Québec. Ceci se passait à l'été de $1658 .{ }^{15}$ Un an plus tard François de Laval, évêque de Pétrée et vicaire apostolique de la Nouvelle-France, débarquait à Québec.

Pour nos historiens, Mgr de Laval, c'est vraiment l'homme de taille à engager le combat avec l'adversaire gallican. Jusqu'ici, les Harlay n'ont rencontré que déférence glaciale, mais point de résistance. On a beau être "romain", il faut quand

14 Faillon, op. cit., 2: 278-279. Se référant à Mgr de Harlay, Faillon traduit "spontanea voluntate" par "de son propre mouvement" en feignant d'ignorer qu'il s'agit d'une expression courante dans les documents officiels signifiant que la décision a été prise en toute liberté.

15 Voir Rochemonteix, op. cit., 2: 217-228. - Le Journal des Jésuites, 220-239, passim. 
même respecter la hiérarchie. Mais avec Mgr de Laval, c'est bien différent, puisque c'est mître contre mître.

Vicaire apostolique, le nouveau prélat relève directement de Rome via la Sacrée Congrégation de la Propagande. Voilà qui au point de départ est rassurant. Mais cela ne s'est pas fait tout seul. Encore ici, les Jésuites ont joué leur rôle. Entre le vicariat apostolique et l'évêché en titre, alternative que Rome proposait, ils ont choisi le premier, après avoir exercé leur pouvoir de persuasion sur Anne d'Autriche. ${ }^{16}$ Mais $\mathrm{Mgr}$ de Harlay qu'on laissait pour compte ne l'entendait pas de cette oreille. Il fit tonner les gros canons gallicans: les parlements de Paris et de Rouen défendirent au vicaire apostolique d'exercer ses fonctions et l'Assemblée du Clergé de France refusa d'autoriser sa consécration avant que des lettres-patentes ne soient émises par la Cour. ${ }^{17}$

Mais la Cour ne sait pas encore très bien sur quel pied danser. Rome a beau jeu de la presser de prendre ses responsabilités et de débouter Mgr de Harlay de ses prétentions, puisque c'est elle, la Cour, qui officiellement a fait la demande d'un vicariat apostolique. ${ }^{18}$ Mais de son côté, Mgr de Harlay n'exige rien moins que d'obliger $\mathrm{Mgr}$ de Laval à prendre de Rouen des pouvoirs de grand vicaire même s'il est officiellement mandaté par Rome, "prétention plus qu'injurieuse au Souverain Pontife", commente avec indignation le biographe Gosselin. ${ }^{19}$

Finalement la Cour choisit un moyen terme. Le vicaire apostolique exercera ses fonctions épiscopales "mais sans préjudice des droits de la juridiction ordinaire" et cela "en atten-

${ }^{16}$ Voir la correspondance de Gueffier, chargé d'affaires de France à Rome, avec Brienne publiée en appendice dans Rochemonteix, op. cit., 2 : 479-501. Voir aussi Archives de la Sacrée Congrégation de la Propagande [ASCP], Actes, $26: 142-145 ; 27: 275-276$.

17 Harlay à Mazarin, 10 novembre 1658, dans Rochemonteix, op. cit., 2: 502-504. - Abbé Thareau à Mazarin, 10 et 20 décembre 1658 dans ibid., 2: 504-508.

18 ASCP, Actes, 27: 275-276, Gueffier à Brienne, 10 décembre 1658, dans Rochemonteix op. cit., 2: 501 .

19 Harley à Mazarin, 3 mars 1659, dans ibid., 2: 510. - Gosselin, Vie de Mgr de Laval (2 vol., Québec, 1890), 1: 131. 
dant l'érection d'un évêché dont le titulaire sera suffragant de l'archevêque de Rouen". ${ }^{20}$ C'est le texte même des lettrespatentes du roi à Mgr de Laval. La fin "gâtait un peu le reste", diront nos historiens. ${ }^{21}$

Mais ce n'est là qu'une minute d'égarement de la part de la Cour. Anne d'Autriche avertit le gouverneur d'Argenson qu'on ne doit souffrir au Canada aucune autre juridiction que celle de Mgr de Laval. ${ }^{22}$ Mais est-ce bien la conversion définitive à l'orthodoxie? Pas tout à fait. Nouvelle minute d'égarement, nouvel accès, bien léger, de gallicanisme, on mande à d'Argenson de veiller à ce que soient appliquées intégralement les lettres-patentes, c'est-à-dire à ce que les deux juridictions celle de Rome et celle de Rouen s'exercent concurremment. ${ }^{23}$ Puis on revient à la droite raison une fois de plus, et le pauvre d'Argenson est prié de tenir la main à ce que l'autorité de $\mathrm{Mgr}$ de Laval s'exerce à l'exclusion de toute autre. ${ }^{24}$

Toujours vicaire de Rouen, M. de Queylus a eu un moment l'espoir qu'il pourrait continuer à assumer ses fonctions. Mais la Cour qui, comme on sait, a le sens de la légitimité, en a décidé autrement après quelques hésitations.

Queylus s'embarque pour la France, mais Mgr de Laval qui a le soupçon aigu, craint qu'il n'aille y tramer, avec $\mathrm{Mgr}$ de Rouen et les Sulpiciens, quelque machination qui mette son autorité en péril. Il s'en ouvre à la Congrégation de la Propagande. ${ }^{25}$ De son côté, le roi qui lui a renouvelé son appui interdit à $M$. de Queylus de quitter le royaume, ce qui n'empêche pas l'obstiné sulpicien de se rendre à Rome et d'obtenir de la Daterie - subrepticement disent nos historiens - une bulle assurant l'érection de la paroisse de Montréal indépendamment de

2027 mars 1659, Archives du Séminaire de Québec [ASQ], Evêque de Québec, no 210.

21 Gosselin, op. cit., $2: 133$.

2231 mars 1659, ASQ, Lettres, Carton N, no 1. IV, no 6 .

2311 mai 1659, voir Le Journal des Jésuites, 264 et ASQ Polygraphie

2414 mai 1659, ASQ, Lettres, Carton N, no 2.

25 AAQ, Copies de Lettres, 1: 21. 
la juridiction exercée par Mgr de Laval. Ce qui est le comble de l'humour noir, l'archevêque de Rouen délègue à Mgr de Laval le pouvoir d'installer M. de Queylus à la nouvelle cure. ${ }^{26}$ "S'imagine-t-on, s'exclame l'historien Gosselin, un évêque de France déléguant un vicaire apostolique dépendant du Saint-Siège pour présider à l'installation d'un curé dans le territoire de ce même vicaire apostolique!" 27

La mesure était pleine, ce qui justifiera bien entendu Mgr de Laval d'utiliser l'arme de la suspense, quand M. de Queylus osera revenir au Canada. Le séjour de celui-ci sera bref cependant, moins de trois mois. Le roi lui intimera l'ordre de quitter la colonie. Et ce sera sans réplique. À la fin de 1661, Mgr de Laval et Rome restaient les seuls maîtres de l'Église du Canada..8

Pourtant l'ombre de Rouen continuait de planer sur la colonie, au moins dans l'imagination de Mgr de Laval qui craignait encore en 1666 que les Messieurs de Saint-Sulpice, avec la bénédiction de Mgr de Harlay, ne s'essaient à nouveau de s'emparer des cures de la colonie. Ces soupçons, ajoutés au fait que les administrateurs civils de la Nouvelle-France tenaient pour peu de chose son titre de vicaire apostolique l'amenèrent à souhaiter ardemment la création d'un évêché en bonne et due forme à Québec et sa nomination comme premier titulaire. C'était aussi, depuis longtemps, le désir du roi. ${ }^{29}$

Tenace, Mgr de Harlay ne mettra pas de temps à réapparaître dans les négociations entreprises auprès de Rome pour obtenir le changement du vicariat apostolique en évêché. Ce qu'il réclame maintenant c'est que le futur évêché soit suffragant de l'archidiocèse de Rouen conformément à ce qu'expri-

26 Louis XIV à Laval, 13 mars 1660. ASQ, Lettres, Carton N, no 4. Louis XIV à Queylus, 27 février 1660. AAQ, Reg. A, 179. Faillon, op. cit., 2: 480. AiSQ, paroisses diverses, no 89 .

27 Gosselin, op. cit., 2 : 195-196.

28 Le Journal des Jésuites, 301, AAQ, Copies de lettres, 1: 27. Reg. A, 140-142. - Rochemonteix, op. cit., 2: 301. - Le Journal des Jésuites, 303.

29 Laval à la Propagande, 13 octobre 1666. AAQ, Copies de Lettres, 1: 49. Laval au pape, 8 novembre 1665, AAQ, Copies de lettres, 1: 47. Louis XIV à Laval, 13 mars 1660. ASQ, Lettres, Carton N, no 4. Louis XIV au pape, 14 décembre 1662, AAQ, Eglise du Canada, VII: 77. 
maient les lettres-patentes délivrées par le roi à Mgr de Laval en 1659. Et cette fois, la Cour de France, prise d'une nouvelle attaque du vieux mal gallican, soutient fermement les prétentions rouennaises..$^{30}$ Même Mgr de Laval, à bout de force et de patience devant les pourparlers qui traînent en longueur, se permet de flancher. Il accepte d'être rattaché et subordonné à son vieil opposant, l'archevêque de Rouen. ${ }^{31}$ C'est une concession qu'on lui arrache, soulignent les historiens, et à laquelle il consent finalement pour un bien supérieur, celui de son Église.

Heureusement, Mgr de Harlay émoussera ses armes contre l'ultime bastion. Rome reste imprenable; les cardinaux de la Consistoriale et le pape, absolument intraitables. Comme pour eux le temps ne compte pas, l'affaire traîne en longueur, puis, le roi finit par abandonner ses exigences et $\mathrm{Mgr}$ de Harlay, transféré du siège de Rouen à celui de Paris, trouvera bien de quoi occuper son énergie à quelques bonnes querelles gallicanes qui se précisent à l'horizon. En 1674, Mgr de Laval est devenu évêque titulaire du diocèse de Québec rattaché directement au Saint-Siège. ${ }^{32}$ L'orthodoxie était sauve. En 1923, le chanoine Groulx écrivait: "François de Laval fut, au sens magnifique du mot, un évêque romain. Il voulut l'être par sa nomination, qu'en sa qualité de vicaire apostolique, il tint de Rome exclusivement; il voulut l'être par le sacre, recevant du Nonce d'Alexandre VII l'onction du pontificat; plus tard, il voulut l'être par l'érection de son diocèse, créé indépendant de tout évêché de France et rattaché immédiatement au Saint-Siège." 33

Le chanoine Groulx ne pouvait guère mieux résumer en aussi peu de mots la thèse de ses devanciers, qui du reste continue à imprégner un certain nombre d'ouvrages récents.

Mgr de Laval, évêque romain, voilà bien le fin mot de l'affaire et qui explique par ricochet pourquoi, les évêques Har-

${ }^{30}$ Louis XIV au pape, 28 juin 1664. ASQ, Séminaire 15, no 7.

31 ASCP, Actes 39: 68-69. AAQ, Copies de Lettres, 1: 85 et 87.

32 Mandements des évêques de Québec, 1ère série (4 vol., Québec 18871888), 1: 82-90. 107-108.

33 Lionel Groulx, Notre Mâ̂tre le Passé, 1ère série (Montréal, 1937), 
lay, M. de Queylus et les sulpiciens de Montréal nous aient été montrés sous un jour si peu flatteur. Car pour nos historiens, ils sont bien moins romains et certainement plus gallicans.

Notre historiographie, en ce qui touche ces problèmes a été bâtie moins par des historiens que par des canonistes. Le fait historique les intéresse moins que le droit, ou mieux, c'est le droit qui donne au fait historique sa signification et son importance. Ils ne se demanderont pas si la conjoncture sociale et religieuse, coloniale et métropolitaine du XVII ${ }^{e}$ siècle pouvait expliquer les prétentions de l'archevêque de Rouen. Ils se demandent plutôt s'il est admissible qu'un évêque de France persiste à vouloir exercer son autorité là où Rome exerce directement la sienne. Car pour eux, le gallicanisme ce n'est pas d'abord une réalité historique, c'est une erreur doctrinale. D'où le peu de sympathie qu'ils manifestent à tous ceux qui paraissent en être atteints et qui, par surcroit, ont tous le mauvais goût de s'attaquer à un personnage qui a le double mérite d'être évêque fondateur et promis aux autels.

Et l'erreur gallicane, ils la considèrent en hommes du $\mathrm{XIX}^{\circ}$ siècle, comme une déviation à un ultramontanisme farouche, fort en vogue à cette époque, mais qui ressemble d'assez loin à ce qui se passait au XVII' ${ }^{e}$ siècle.

Dans la France d'alors, le gallicanisme c'était fondamentalement une sorte de nationalisme religieux dont tout le monde était plus ou moins atteint $\mathrm{y}$ compris ceux qu'on qualifie d'anti-gallicans parce qu'ils refusaient de pousser jusqu'à leur extrême limite les privilèges dont était nantie l'Église de France.

Aussi le portrait qu'on nous a laissé de Mgr de Laval est-il, à cet égard, celui d'un homme vivant dans un autre milieu et dans un autre âge, peut-être bien justement le milieu canadienfrançais du XIX $\mathrm{X}^{\bullet}$ siècle. Deux petits faits aident cependant à le resituer à son époque véritable. En 1674, selon le témoignage d'un ami intime Michel Gazil, l'homme, qu'on nous donne com- 
me un défenseur acharné de l'autorité pontificale, se plaint qu'on ait dans la demande de sa bulle négligé les privilèges gallicans. Gazil devra expliquer que le texte de la bulle a été réformé et corrigé par des juristes gallicans "de sorte que, dit-il, l'on n'a rien laissé en icelle bulle qui fut préjudiciable aux avantages de la France". Puis il ajoute, "l'Église de Québec ne sera pas plus sujette aux dispositions extraordinaires de la Cour de Rome que toutes nos métropoles de France qui relèvent immédiatement de Rome comme sont Lyon, Rouen, Bourges". ${ }^{34}$

Trois ans plus tard, M. Dudouyt, agent de l'évêque de Québec à Paris, raconte à Mgr de Laval une entrevue qu'il a obtenue du ministre Colbert. Je lui ai dit, confie-t-il, "qu'il était vrai que l'évêché de Québec relevait immédiatement du Saint-Siège mais que vous aviez toujours témoigné qu'il relevrait de tel archevêché de France qu'il plairait au roi et que dès il $\mathrm{y}$ a plus de dix ans, M. de Rouen vous ayant dit qu'il demanderait qu'il relevât de son archevêché, que vous lui aviez dit que vous en étiez content si le roi le voulait agréer. Il dit (il s'agit de Colbert) que c'était une chose contre les droits du Royaume de le faire relever immédiatement du Saint-Siège, que le roi ne le souffrirait pas, qu'il donnerait des lettres de déclaration sur vos bulles, qu'il faudrait les faire registrer au parlement, qu'il les donnerait en la forme qu'il faudrait. Je lui ai dit que vous les recevriez en telle manière que le roi voudrait les faire expédier, et comme je savais qu'on était résolu de le faire relever de Paris, je l'ai demandé à M. Colbert afin que l'on se conforme dans la conduite ecclésiastique à la coutume et à l'usage de Paris comme on fait de la conduite civile." Puis Dudouyt termine en disant: "Cela donnera lieu à l'archevêque de Paris de protéger l'Église du Canada." 35

Mais protéger l'Église du Canada contre quoi. Contre des abus de pouvoir possibles du Saint-Siège. Et quel est ce person-

${ }^{34}$ Gazil à Pallu, 24 juillet 1674, dans Altera Nova Positio super Virtutibus, Quebecen Beatificationis et Canonizationis ven. servi dei Francisci De Montmorency-Laval Primi Episcopi Quebecensis (Sacra Rituum Congregatione, 1958), Doc XLV, 4:340.

${ }^{85}$ Dudouyt à Laval, 1677, ASQ, Lettres, Carton N, no 48. 
nage puissant dont Mgr de Laval, par son agent à Paris, sollicite la protection: nul autre que François de Harlay, ex-archevêque de Rouen et maintenant archevêque de Paris.

Comme on le voit, il faut mettre certaines réserves importantes aux tendances romaines de Mgr de Laval. Et tout compte fait, il n'est pas du tout sûr qu'il ait été d'esprit moins gallican que les évêques Harlay et $M$. de Queylus. Mais alors comment expliquer les différends qui les ont opposés? Revoyons brièvement les choses.

L'archevêque de Rouen prend pied au Canada par le biais de l'autorité qu'il exerce sur les Hospitalières de Dieppe qui viennent s'installer à Québec. Le fait est déjà significatif. Les Hospitalières forment une institution déjà imbriquée dans les cadres de l'Église de France, avec des cellules soumises à la juridiction de l'Ordinaire sur le territoire duquel elles se trouvent. Si quelques religieuses de Dieppe décident d'immigrer au Canada, elles le feront avec la permission de leur évêque et resteront vraisemblablement sous son autorité tant qu'un pouvoir épiscopal n'existera pas dans la colonie. Pourquoi? Parce que dans un contexte gallican, il eut été impensable qu'on abandonne à la juridiction romaine, représentée par les Jésuites, des religieuses françaises déjà intégrées à l'Église de France. Mais comment expliquer la situation des Jésuites eux-mêmes? Les Jésuites sont au Canada comme missionnaires et comme tels, ils relèvent de Rome. $\mathrm{Au} \mathrm{XVII}{ }^{\mathrm{e}}$ siècle, l'évangélisation des infidèles est sans doute la seule forme d'apostolat qu'on abandonne, et encore de mauvaise grâce, à la centralisation romaine. De mauvaise grâce, car il se trouve bien des gens en France pour demander que les missions soient aussi du ressort de l'Église nationale, groupant séculiers et laïques. Les Véritables Motifs des Messieurs et Dames de la Société de Notre-Dame de Montréal en sont un bon exemple.

En 1647 des lettres venues de France mettent en doute l'autorité des Jésuites à recevoir les professions religieuses. De quelles professions religieuses peut-il s'agir? Evidemment de celles des Ursulines. Contrairement aux Hospitalières, les Ursu- 
lines sont venues de plusieurs régions de France et en principe elles restent soumises à l'Ordinaire de leur diocèse d'origine. Que chacun de ces évêques délègue son autorité au Supérieur des Jésuites de Québec constituerait une procédure assez lourde. D'autre part nantis des seuls pouvoirs romains, les Jésuites ne sont pas habilités aux yeux du monde français, à présider aux "vestures", comme dit le texte du Journal. La solution, c'est de se rattacher officiellement à un évêque de France. Lequel? Celui de Rouen, sans doute parce qu'il est déjà dans la place. ${ }^{36}$

Le fait est d'une importance notable. C'est à ce moment, l'année 1648 précisément, que s'établissent les premières structures de l'Église du Canada, orientées en fonction des besoins de la population française de la colonie. Car il s'agit bien essentiellement de ces Français, même s'ils sont peu nombreux, sur lesquels l'Église gallicane entend maintenir ses droits. Et ce qu'on met en doute ce n'est pas seulement la capacité des Jésuites à recevoir les professions religieuses, c'est même la validité des mariages qu'ils bénissent.

Ces nouvelles structures de 1648 conçues pour les Français catholiques de la colonie s'installent parallèlement aux structures de l'Église missionnaire qui existent depuis 1632, qui relèvent de Rome et qui ont été mises sur pied pour les besoins d'une population infidèle. En fait, c'est une réalité coloniale, bien particulière, c'est-à-dire la naissance d'une colonie de peuplement au cœur même d'une terre de mission, qui oblige à ce partage des juridictions. Que ces juridictions se soient établies dans ce qui semble une sorte d'empirisme s'explique d'une part par le fait que l'expansion coloniale n'avait pas été prévue par le droit gallican, et d'autre part par le fait que le droit missionnaire en était encore à ses débuts, d'ailleurs pénibles, où la Congrégation de la Propagande avait à affronter ces impérialismes redoutables que furent les patronats portugais $\mathrm{e}^{\hat{t}}$ espagnols.

36 Pour le statut des Ursulines, voir Dom Jamet, éd., Marie de l'Incarnation - Ecrits spirituels et historiques (4 vol., Paris et Québec, 19291939), 3: 123, 390-392; 4: 19-21, 58-62, 109, 264-265. — Les Ursulines de Québec (4 vol., Québec, 1863), 1: 69, 71, 135. 
Le silence des Jésuites sur les pouvoirs qu'ils détiennent de Rouen, de 1648 à 1653, devient plausible justement parce que le droit de Mgr de Harlay est mal établi non pas vis-à-vis Rome, mais vis-à-vis les autres évêques de France. ${ }^{37}$ En somme, pourquoi l'archidiocèse de Rouen plus qu'un autre. Il semble bien ici que c'est le temps de la possession effective qui tiendra lieu de droit.

Dans l'optique que nous tentons de dégager, la querelle qui oppose M. de Queylus aux Jésuites paraît être une querelle de subalternes ou de subalternes frustrés, accentuée par la rivalité d'ordres religieux différents et surtout par l'opposition Monttréal-Québec qui existe de façon tenace depuis 1642. Mais il y a un élément positif dans tout cela, c'est que M. de Queylus représente pour la première fois l'établissement des cadres séculiers à l'intérieur de l'Église dominée par Mgr de Harlay.

Quand en 1658, celui-ci pour régler le conflit décide de diviser sa juridiction entre Montréal et Québec, c'est une mesure provisoire qu'il prend en attendant la nomination imminente d'un évêque qui selon ses vues ne saurait être qu'un évêque titulaire, et selon ses souhaits, qu'un évêque suffragant de son propre archidiocèse.

Mais c'est un vicaire apostolique qui finalement est nommé. Les Jésuites ne sont pas étrangers à ce changement, mais peu importe ici. Ce qui importe c'est de savoir ce qu'est un vicariat apostolique aux yeux d'un gallican. Ce ne peut-être que le perfectionnement, que le couronnement des structures d'une Eglise missionnaire. Par conséquent, cela ne touche en rien à la juridiction gallicane déjà établie. Aussi la proposition de Mgr de Harlay voulant que Mgr de Laval prenne de lui des pouvoirs de grand vicaire n'a-t-elle rien d'irrespectueux pour l'autorité du Saint-Père.

D'ailleurs les lettres-patentes émises par la Cour sur les bulles de Mgr de Laval correspondent exactement à l'état des

${ }^{37} \mathrm{Il}$ est clair que les Jésuites tout en voulant être en règle ne tiennent pas à s'attirer des ennuis. D'ailleurs ils ne sont pas sans savoir que dans les cadres de l'Eglise gallicane leur rôle comme desservants de la population française ne peut être que supplétif et provisoire. 
structures de l'Église du Canada en 1659 et à l'idée qu'on se faisait en France des pouvoirs d'un vicaire apostolique rattaché à la Congrégation de la Propagande. Mgr de Laval exercera sa juridiction à l'intérieur des cadres de l'Église missionnaire, mais sans préjudice de la juridiction ordinaire détenue par l'archevêque de Rouen.

Dès lors, l'affaire Laval-Queylus prend un tout autre sens. C'est une juridiction établie depuis plus de dix ans et reconnue par les lettres-patentes de 1659 que le vicaire général de Rouen défend contre le vicaire apostolique de Rome, ou si l'on veut, c'est l'Église des colons qu'il défend contre l'impérialisme menaçant de l'Église des missions.

Et si à la fin on ne lui donne pas raison, ce n'est pas qu'on reconnaît la primauté du droit de Rome. C'est que le roi de France, chef de l'Église gallicane, en a décidé autrement. Pourquoi ? Sans doute parce que si au plan des structures deux juridictions peuvent se défendre, en pratique cela donne lieu à des conflits interminables. Les dernières années de la vie religieuse en Nouvelle-France étaient là pour le prouver. Mais pourquoi alors avoir choisi Mgr de Laval de préférence à $M$. de Queylus. Le vicaire apostolique avait sur le grand vicaire de Rouen l'avantage d'être évêque, dignité dont on ne pouvait se passer dans la colonie. Mais surtout, dès 1660 , ce vicaire apostolique était devenu aux yeux du roi l'évêque en titre de Québec et il était d'ores et déjà destiné à unifier les deux orientations qu'avait prises l'Église du Canada avant son arrivée. Mgr de Laval à partir de 1663, ne dédaignera jamais de se déclarer "évêque de Pétrée, nommé par le roi évêque de Québec".

De fait, c'est en véritable évêque français et gallican au sens profond du terme qu'ils édifiera son Église en 1663 à 1674. Sans doute il s'est inspiré d'une philosophie missionnaire ce n'est pas pour rien que les Jésuites l'avaient choisi - mais il soumettra chacun de ses gestes à la Cour de France, non à Rome, pour approbation.

Restait l'érection officielle du diocèse de Québec. Pour Louis XIV, ce ne pouvait être qu'une formalité. Quand en Fran- 
ce on souhaite que le futur évêché soit rattaché à la métropole de Rouen, on reste logique avec soi-même. Quand Mgr de Laval accepte d'être suffragant de Mgr de Harlay, c'est qu'il sait que, contrairement à la situation de 1659 , son autorité n'est plus en cause en Nouvelle-France, puisque, de Versailles et de Rome, il a l'assurance qu'il sera le titulaire du nouvel évêché. En somme, ce que veulent les intéressés dans la colonie comme dans la métropole, c'est parfaire les cadres de l'Église du Canada pour qu'elle s'imbrique dans la structure hiérarchique de l'Église gallicane.

Mais Rome ne se laisse pas facilement convaincre. En fait, pendant dix ans, l'érection du diocèse de Québec deviendra le prétexte insignifiant d'une lutte titanesque de prestige entre Rome et Versailles.

Versailles finira par abandonner la partie quand les principaux intéressés (dont Mgr de Laval) se seront laissés convaincre que le jeu n'en valait pas la chandelle et que les privilèges gallicans avaient autant de chance d'être respectés si Québec était rattaché à Rome plutôt qu'à Rouen ou une autre métropole française. En quoi, la suite de l'histoire prouvera qu'on avait vu juste.

Vu sous cet angle qui met l'accent sur le fait historique plutôt que sur le droit, sur la compréhension plutôt que sur le jugement, l'élaboration des structures de l'Église du Canada jusqu'en 1674 nous apparaît assez différente de celle à laquelle l'historiographie traditionnelle nous avait habitués.

Ces structures c'est à mon sens le point essentiel. Mais pour ceux qui ont un faible pour les hommes, il leur reste aussi le loisir de réaménager la galerie des fondateurs de l'Église du Canada et d'y mettre un peu plus en évidence Messeigneurs de Harlay et M. de Queylus.

JEAN BLAIN

Département d'Histoire

Université de Montréal 